\title{
ACTIVIDAD HIDROLÍTICA DE AISLADOS BACTERIANOS CON POTENCIAL APLICACIÓN EN EL TRATAMIENTO DE EFLUENTES DE FRIGORÍFICO
}

\section{HYDROLYTIC ACTIVITY OF BACTERIAL ISOLATES WITH POTENTIAL APPLICATION FOR TREATMENT OF SLAUGHTERHOUSE EFFLUENTS}

\author{
RODAS M. ELKIN, ${ }^{1,2}$ M.Sc, BOTELLO S. WILMAR, ${ }^{1,2 *}$ Ing. \\ 'Universidad Estadual Paulista, Jaboticabal (UNESP),Facultad de Ciencias Agrarias y Veterinarias, SP, Brasil. \\ ${ }^{2}$ Fundación Universitaria de San Gil-Facultad de Ciencias Naturales e Ingeniería. Yopal, Colombia.
}

\section{Key words:}

hydrolytic bacteria, extracellular enzymes, treatment of slaughterhouse wastewater.

\section{Abstract}

Slaughterhouse effluents are characterized by high concentrations of proteins, fats and polysaccharides. The degradation of these compounds to their monomeric constituents is performed by the action of extracellular enzymes produced and secreted by hydrolytic bacteria (HB). Accordingly, the activity of these microorganisms increased the degradation rates of the organic fraction present in this waste. In the present study, a total of 15 bacteria producing extracellular enzymes were isolated and selected to provide a rate of enzymatic activity $\geq 2.0$. Of these 15 isolates, 7 had proteolytic activity (46.6\%), 3 amylolytic $(20 \%), 3$ cellulolytic (20\%) and 2 lipolytic (13.4\%). Additionally, antagonistic effect was evidenced among some of the selected isolates, indicating a possible competitive disadvantage under natural conditions. We conclude that the HB obtained show a promising potential for optimizing treatment systems waste water from the slaughterhouse industry.

\section{Resumen}

Los efluentes de la industria frigorífica se caracterizan por presentar altas concentraciones de proteínas, grasas y polisacáridos. La degradación de estos compuestos a sus constituyentes monoméricos es realizada por la acción de enzimas extracelulares, producidas y secretadas por bacterias hidrolíticas (BH). En consecuencia, la actividad de estos microorganismos favorece el aumento de las tasas de degradación de la fracción orgánica presente en este tipo de residuos. En el presente estudio, un total de 15 bacterias productoras de enzimas extracelulares fueron aisladas y seleccionadas al presentar un índice de actividad enzimática $\geq$ 2,0. De estos 15 aislados, 7 presentaron actividad proteolítica (46,6\%), 3 amilolítica $(20 \%), 3$ celulolítica $(20 \%)$ y 2 lipolítica $(13,4 \%)$. Adicionalmente, fue evidenciado efecto antagónico entre algunos de los aislados seleccionados, indicando su posible desventaja competitiva en condiciones naturales. Se concluye que las $\mathrm{BH}$ obtenidas evidencian un potencial promisorio para la optimización de sistemas de tratamiento de aguas residuales procedentes de la industria frigorífica. 


\section{Introducción}

Los efluentes generados en la industria frigorífica son típicamente tratados mediante sistemas que involucran operaciones físicas, químicas y biológicas (MUÑOZ 2005; PABÓN y GÉLVEZ, 2009; LIU et al., 2015). Estos procesos tienen la finalidad de dar cumplimiento a las directrices establecidas por la normatividad ambiental, y garantizar que los efluentes tratados puedan ser vertidos en sistemas hídricos receptores sin generar riesgos a nivel ambiental y sanitario. Dada la composición característica de los efluentes residuales de frigorífico, representada principalmente por la presencia de altas concentraciones de grasas, proteínas y polisacáridos (PALATSI et al., 2011), la fase biológica es de especial interés para la estabilización de este tipo de residuo. En dicha etapa es aprovechada la actividad metabólica microbiana para reducir la concentración de materia orgánica presente (CHAN et al., 2009). El proceso inicia con el fraccionamiento (hidrólisis) de biopolímeros hasta sus constituyentes monoméricos (como por ejemplo aminoácidos, ácidos grasos y monosacáridos), lo cual se logra por acción de enzimas extracelulares denominadas hidrolasas (CUNHA et al., 2010). Estas enzimas son secretadas al medio por bacterias hidrolíticas $(\mathrm{BH})$, las cuales se pueden clasificar en tres categorias: 1) bacterias proteolíticas, productoras de proteasas; 2) bacterias sacarolíticas, productoras de celulasas y amilasas; y 3) bacterias lipolíticas, productoras de lipasas (GERARDI, 2003). La actividad concertada de estas bacterias permite la generación de moléculas solubles, que pueden ser utilizadas como fuente de carbono y energía y constituyen un sustrato biodisponible para otro tipo de comunidades microbianas presentes, dando continuidad al proceso de degradación y estabilización (ARNOSTI, 2011). La hidrólisis, en consecuencia, constituye una fase crítica y limitante de la tasa de degradación de efluentes con alta carga orgánica, y el éxito del proceso de tratamiento está estrechamente relacionado con el crecimiento, estabilidad y potencial de las BH (VAVILIN et al., 2008; FACCHIN et al., 2013).

Un enfoque que puede tener gran potencial para aumentar la eficiencia de la fase hidrolítica es la inoculación de microorganismos que hayan sido aislados y seleccionados para el tratamiento de compuestos específicos presentes en aguas residuales (VAN DER GAST et al., 2004; HERRERO y STUCKEY, 2014). La adición de aislados bacterianos con actividades metabólicas específicas recibe el nombre de bioaumentación (HERRERO y STUCKEY, 2014). Al respecto, los microorganismos utilizados en este proceso deben ser eficientes en la degradación de contaminantes, además de ser compatibles, competitivos y persistentes con las comunidades nativas presentes en dichos sistemas de tratamiento (YU y MOHN, 2002).
Diversos estudios han analizado el efecto de la incorporación de consorcios de $\mathrm{BH}$ en sistemas de tratamiento de efluentes industriales con diferente grado de éxito (MOHAN et al., 2005: ČATER et al., 2015; MARTIN-RYALS et al., 2015). Dichos estudios ponen de manifiesto a nivel experimental, que la actividad de estos microorganismos puede conferir ventajas en la partida de sistemas de tratamiento, aumento de las eficiencias de remoción y mejor recuperación de gases de interés energético (como por ejemplo hidrógeno y metano) generados en los procesos de estabilización anaerobia (CAVALEIRO et al., 2010; MARONE et al., 2012).

No obstante, es indispensable garantizar que dichos inoculantes presenten elevado potencial hidrolítico y que puedan permanecer y establecerse en el sistema de tal forma que se garantice su actividad y viabilidad. La alteración de estos factores puede ocasionar una escasa o nula eficiencia del proceso de incorporación de microorganismos al sistema, debido a la falta de especies bacterianas con rutas metabólicas claves en dicha transformación (HERRERO y STUCKEY 2014). En este estudio fue realizado el aislamiento de bacterias productoras de enzimas extracelulares (proteasas, lipasas, celulasas y amilasas), asociadas a un sistema de tratamiento de aguas residuales de frigorífico. Fue estimada la actividad hidrolítica y el efecto antagónico de cada uno de los aislados seleccionados. Este trabajo constituye un primer paso para la generación de un consorcio bacteriano altamente eficiente en la degradación la fracción orgánica de efluentes procedentes de industrias frigoríficas.

\section{Materiales y Métodos}

Muestreo y obtención de aislados bacterianos. Para la colección de la muestra fue seleccionado un sistema de tratamiento de aguas residuales establecido en el Frigorífico "La Frontera", ubicado en el municipio de Villa del Rosario, Norte de Santander, Colombia. Fue colectada una muestra de $500 \mathrm{ml}$ de lodos activados contenidos en un reactor aerobio. La recuperación de aislados bacterianos con características hidrolíticas fue realizada mediante el establecimiento de diluciones seriadas y siembra en superficie en los siguientes medios de cultivo: a) Plate count suplementado con leche descremada al $1 \% \mathrm{p} / \mathrm{v}$ para la selección de bacterias actividad proteolítica (BAP); b) Medio mineral con la siguiente composición $(\mathrm{g} / \mathrm{l}):\left(\mathrm{NH}_{4}\right)_{2} \mathrm{SO}_{4}$ 3.2; $\mathrm{K}_{2} \mathrm{HPO}_{4} 2.0 ; \mathrm{KH}_{2} \mathrm{PO}_{4} 1.2 ; \mathrm{MgSO}_{4} 7 \mathrm{H}_{2} \mathrm{O} 0.5$; $\mathrm{CaCl}_{2}$ 0.2; $\mathrm{NaCl} 0.2$; agar-agar 20.0. El medio fue suplementado con $1 \%$ de almidón soluble o con $1 \%$ de carboximetilcelulosa como única fuente de carbono, para la selección de bacterias con actividad amilolítica (BAA) o celulolítica (BAC), respectivamente; y c) Agar tributirina $(\mathrm{g} / \mathrm{l}): 1,3 \%$ caldo nutritivo, $1 \%$ tributirina y $2 \%$ 
agar-agar para el aislamiento de bacterias con actividad lipolítica (BAL). (BEN-GIGIREY et al., 2000). Las condiciones de incubación fueron de $48 \mathrm{~h}$ a $37^{\circ} \mathrm{C}$ para el aislamiento de BAP, BAA y BAL y de 7 días a $37^{\circ} \mathrm{C}$ para la recuperación de BAC. La selección de los aislados fue realizada a partir de las colonias que mostraron halos de hidrólisis luego del periodo de incubación (tanto para la actividad proteolítica y lipolítica), o su crecimiento en los medios de cultivo, en el caso de aislados con posible potencial amilolítico y celulolítico.

Actividad hidrolítica. La estimación de la actividad hidrolítica fue realizada de manera semicuantitativa empleando el índice enzimático (IE). Este índice correlaciona el diámetro de los halos de hidrolisis (Dh) con el diámetro de la colonia (Dc), y fue estimado mediante la siguiente ecuación: IE= Dh/Dc (HANKIN et al., 1975). Según LEALEM y GASHE (1994), microorganismos con una eficiente producción de enzimas extracelulares presentan IE $\geq 2,0$. Teniendo en cuenta este criterio fue realizada la selección $\mathrm{BH}$ con mayor producción de enzimas extracelulares. Para ello, cultivos axénicos de cada uno de los aislados fueron inoculados sobre los medios establecidos en la etapa anterior. Las pruebas fueron realizadas por triplicado y las condiciones de incubación fueron de $37^{\circ} \mathrm{C}$ por 48 horas. En el caso de la actividad amilolítica, los halos de hidrólisis fueron revelados con la adición de $5 \mathrm{ml}$ de lugol después del periodo de incubación, y para la estimación de la actividad celulolítica, los halos de hidrólisis fueron revelados mediante la técnica de coloración con rojo congo (TEATHER, M. et al, 1982).

Caracterización de los aislados seleccionados y efecto antagónico. Las $\mathrm{BH}$ que presentaron IE $\geq 2,0$ fueron analizadas mediante la técnica de tinción de Gram, con la finalidad de establecer su morfología y tipo de pared celular. Adicionalmente, fue establecido su efecto antagónico mediante una variación del método descrito por ÇADIRCI y ÇITAK (2005). Discos de papel de filtro estériles de $0,5 \mathrm{~cm}$ de diámetro (sensidiscos) fueron impregnados con cada uno de los aislados a evaluar (cultivados previamente $12 \mathrm{~h}$ en caldo nutritivo a $37^{\circ} \mathrm{C}$ ). Los discos inoculados fueron dispuestos sobre placas conteniendo agar nutritivo previamente inoculadas de forma masiva con cada uno de los aislados a evaluar, de tal forma que se enfrentaran entre sí cada uno de los aislados seleccionados. Todos los cultivos empleados fueron ajustados al estándar 0,5 de turbidez de la escala de MacFarland (KONEMAN et al., 2008). El efecto antagónico se evaluó midiendo el diámetro de cada uno de los halos alrededor de los sensidiscos. La presencia de un halo de inhibición $\geq 5 \mathrm{~mm}$ fue considerada como actividad antagónica positiva.

\section{Resultados}

Aislamiento y selección de bacterias con actividad hidrolítica. En la Fig. 1 se indica el número total de $\mathrm{BH}$ seleccionadas en relación con el número de $\mathrm{BH}$ con $\mathrm{IE}$ $\geq 2,0$ para cada uno de los sustratos evaluados. Puede evidenciarse una mayor frecuencia de selección de bacterias proteolíticas $(32,7 \%)$, seguida por amilolíticas $(27,3 \%)$, celulolíticas $(21,8 \%)$ y lipolíticas $(18,2 \%)$. De estos aislados iniciales, un total de 15 presentaron un IE $\geq 2,0$, distribuidos de la siguiente manera: 7 con actividad proteolítica $(46,6 \%), 3$ amilolítica (20\%), 3 celulolítica $(20 \%)$ y 2 lipolítica $(13,4 \%)$.

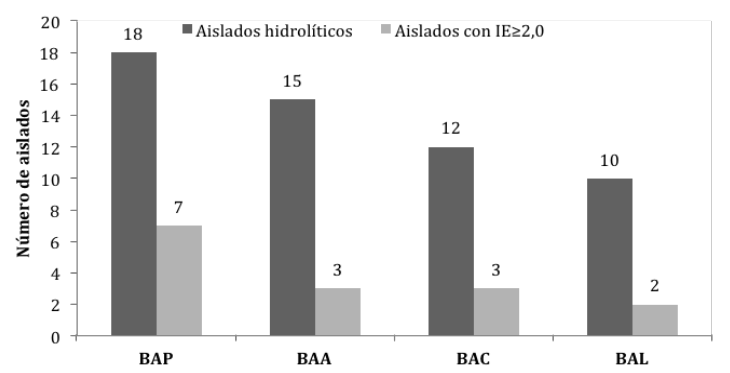

Figura 1. Número total de $\mathrm{BH}$ obtenidas para cada uno de los sustrataos evaluados. BAP: Bacterias con actividad proteolitica; BAA: Bacterias con actividad amilolítica; BAC: Bacterias con actividad celulolítica; BAL: Bacterias con actividad lipolítica. IE: Índice enzimático.

Caracterización de los aislados bacterianos productores de enzimas hidrolíticas. En la Tabla 1 se relaciona la caracterización y los IE estimados para cada uno de los aislados seleccionados. De acuerdo con estos

Tabla 1. Actividad semicuantitativa de hidrolasas producidas por los aislados seleccionados.

\begin{tabular}{ccccccc}
\hline Actividad & Aislado & Morfología & Gram & $\mathbf{D h}^{1}(\mathbf{m m})$ & $\mathbf{D c}^{2}(\mathbf{m m})$ & $\mathbf{I E}^{3}$ \\
\hline \multirow{6}{*}{ Proteolíca } & AP1 & Bacilo & + & 10 & 3 & 3,3 \\
& AP3 & Bacilo & + & 10 & 3 & 3,3 \\
& AP4 & Bacilo & - & 21 & 5 & 4,2 \\
& AP6 & Coco & + & 13 & 3 & 4,3 \\
& AP10 & Bacilo & - & 15 & 5 & 3,0 \\
& AP15 & Bacilo & + & 18 & 4 & 4,5 \\
& AA5 & Bacilo & - & 12 & 5 & 2,4 \\
& AA9 & Bacilo & + & 17 & 5 & 3,4 \\
& AA11 & Bacilo & + & 13 & 3 & 4,3 \\
& AC1 & Bacilo & - & 12 & 3 & 4 \\
Celulolítica & AC4 & Bacilo & + & 13 & 5 & 2,6 \\
& AC5 & Bacilo & + & 12 & 5 & 2,4 \\
& AL1 & Bacilo & + & 20 & 5 & 4,0 \\
& AL2 & Bacilo & + & 15 & 5 & 3,0 \\
\hline
\end{tabular}

${ }^{1} \mathrm{Dh}$ : Diámetro del halo de degradación; ${ }^{2} \mathrm{Dc}$ : Diámetro de la colonia; ${ }^{3} \mathrm{E}$ : Índice enzimático (IE=Dh/Dc). 
resultados, de los 15 aislados obtenidos 11 fueron identificados como gram positivos $(73,3 \%)$ y 4 como gram negativos $(26,7 \%)$, predominando en la mayoría de ellos la morfología bacilar (80\%). Los aislados AP13, AA9, AC1 y AL1 presentaron los mayores IE en cada uno de los grupos enzimáticos evaluados con valores de $4,5,4,3,4,0$ y 4,0 respectivamente (Tabla 1 , Fig. 2 ).

Efecto Antagónico. Esta prueba se realizó con el fin de establecer la compatibilidad entre los aislados bacterianos seleccionados. En la Tabla 2 se relaciona el efecto antagónico presente entre las $\mathrm{BH}$ seleccionadas una vez fueron confrontadas entre sí. Como puede observarse, AA11 presentó clara actividad antagónica sobre AP6, AP13, AA9 y AC4. Estos resultados sugieren que la susceptibilidad presentada por dichos aislados representaría una posible desventaja competitiva en programas de bioaumentación, debido a la incompatibilidad que presentan con los otros aislados bacterianos.

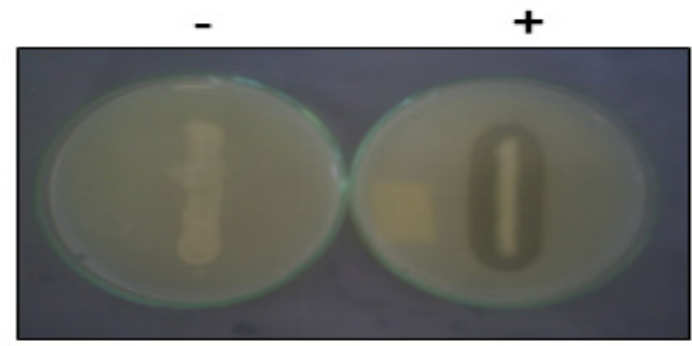

(a)

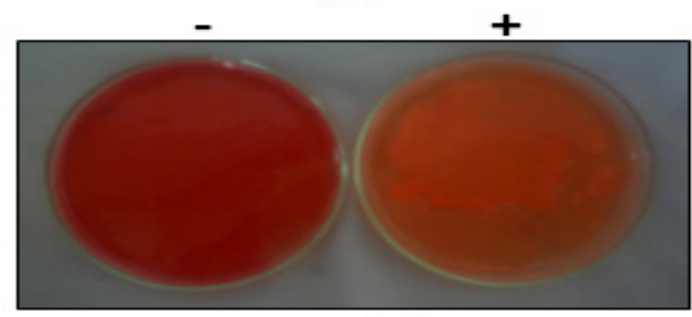

(c)

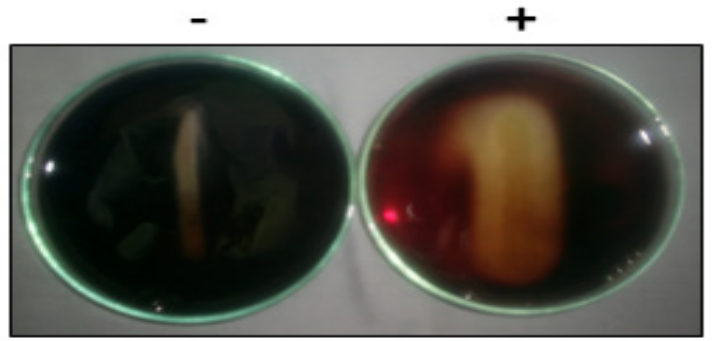

(b)

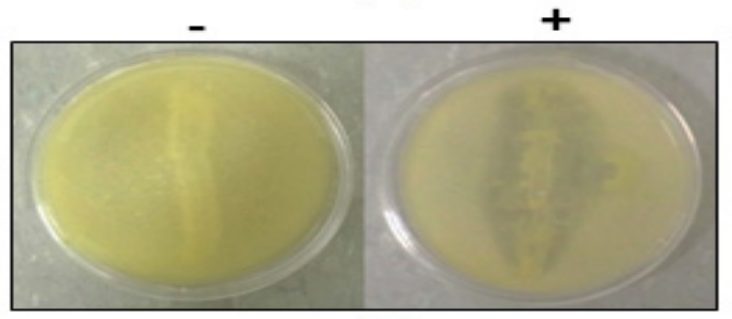

(d)

Figura 2 Detección de halos de hidrólisis en BH con mayor IE para cada uno los sustratos evaluados. a) Actividad proteolitica (Prueba positiva: Aislado AP13); b) Actividad amilolítica (Prueba positiva: aislado AA9) ; c) Actividad celulolítica (Prueba positiva: aislado AC1); d) Actividad lipolítica (Prueba positiva: aislado AL1).

Tabla 2. Efecto antagónico establecido entre los aislados seleccionados.

\begin{tabular}{|c|c|c|c|c|c|c|c|c|c|c|c|c|c|c|c|c|}
\hline \multirow{2}{*}{\multicolumn{2}{|c|}{ Aislado }} & \multicolumn{15}{|c|}{ Testigo $^{2}$} \\
\hline & & $\bar{Q}$ & m & $\frac{\pi}{\frac{\pi}{4}}$ & $\begin{array}{l}0 \\
\frac{1}{<}\end{array}$ & $\frac{0}{\frac{1}{\alpha}}$ & $\frac{m}{\frac{m}{\alpha}}$ & $\frac{10}{\frac{10}{\alpha}}$ & $\sum_{4}^{20}$ & 是 & $\underset{\&}{\frac{5}{8}}$ & $\bar{U}$ & ষ্ঠ & 录 & $\bar{z}$ & $\frac{N}{<}$ \\
\hline \multirow{15}{*}{ 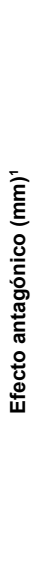 } & AP1 & - & 0 & 0 & 0 & 0 & 0 & 0 & 0 & 0 & 0 & 0 & 0 & 0 & 0 & 0 \\
\hline & AP3 & 0 & - & 0 & 0 & 0 & 0 & 0 & 0 & 0 & 0 & 0 & 0 & 0 & 0 & 0 \\
\hline & AP4 & 0 & 0 & - & 0 & 0 & 0 & 0 & 0 & 0 & 0 & 0 & 0 & 0 & 0 & 0 \\
\hline & AP6 & 0 & 0 & 0 & - & 0 & 0 & 0 & 0 & 0 & 0 & 0 & 0 & 0 & 0 & 2 \\
\hline & AP10 & 4 & 3 & 0 & 0 & - & 0 & 0 & 1 & 3 & 0 & 0 & 0 & 0 & 0 & 0 \\
\hline & AP13 & 0 & 0 & 0 & 2 & 0 & - & 0 & 0 & 0 & 0 & 0 & 0 & 0 & 0 & 0 \\
\hline & AP15 & 1 & 2 & 0 & 3 & 0 & 0 & - & 0 & 0 & 0 & 0 & 2 & 1 & 1 & 0 \\
\hline & AA5 & 1 & 0 & 0 & 1 & 0 & 4 & 0 & - & 0 & 0 & 0 & 2 & 2 & 0 & 3 \\
\hline & AA9 & 0 & 0 & 0 & 0 & 0 & 4 & 0 & 0 & - & 0 & 0 & 0 & 0 & 0 & 2 \\
\hline & AA11 & 1 & 0 & 0 & 7 & 0 & 6 & 0 & 0 & 5 & - & 0 & 6 & 0 & 2 & 0 \\
\hline & $\mathrm{AC} 1$ & 1 & 4 & 0 & 0 & 0 & 0 & 0 & 0 & 0 & 0 & - & 0 & 0 & 0 & 0 \\
\hline & AC4 & 0 & 0 & 0 & 1 & 0 & 0 & 0 & 0 & 0 & 0 & 0 & - & 0 & 0 & 0 \\
\hline & AC5 & 1 & 1 & 0 & 0 & 0 & 0 & 0 & 1 & 2 & 0 & 0 & 0 & - & 0 & 1 \\
\hline & AL1 & 0 & 0 & 0 & 2 & 0 & 1 & 0 & 0 & 0 & 2 & 2 & 0 & 0 & - & 0 \\
\hline & AL2 & 0 & 1 & 0 & 0 & 0 & 0 & 0 & 0 & 3 & 0 & 0 & 1 & 0 & 0 & - \\
\hline
\end{tabular}

${ }^{1}$ El efecto antagónico fue determinado a través de la medición de los halos de inhibición de crecimiento (Dhi) del aislado testigo (mm); ${ }^{2} \mathrm{El}$ testigo de cada prueba corresponde a cada aislado inoculado de forma masiva sobre el medio, confrontado con el total de aislados evaluados. Valores en rojo corresponden a Dhi $\geq 5 \mathrm{~mm}$. 


\section{Discusión}

En este estudio fueron aisladas y seleccionadas bacterias con alta producción de enzimas extracelulares. El criterio utilizado para la selección de estas bacterias se basó en la determinación del índice de actividad enzimática (IE $\geq 2,0)$ (LEALEM Y GASHE, 1994). De los 15 aislados obtenidos, 7 presentaron actividad proteolítica (46,6\%), 3 amilolítica (20\%), 3 celulolítica (20\%) y 2 lipolítica $(13,4 \%)$. La frecuencia de recuperación de un mayor porcentaje de microorganismos proteolíticos se correlaciona probablemente con un mayor contenido de proteínas en este tipo de aguas residuales (PALATSI et al., 2011). No obstante, la aparente baja proporción de bacterias lipolíticas detectada $(13,4 \%)$ podría ser un indicativo de futuras reducciones en la eficiencia del proceso biológico ocasionadas por acumulación de grasa (PERLE et al., 1995). Este fenómeno ya ha sido reportado en procesos biológicos para tratamiento de aguas aeróbicos y anaeróbicos (PERLE et al., 1995; LEFEBVRE et al., 1998; VIDAL et al., 2000). En este aspecto, estrategias de bioaumentación con consorcios microbianos lipolíticos han sido establecidas de manera satisfactoria para el tratamiento de aguas residuales con elevadas concentraciones de grasa (AZHDARPOOR et al., 2014). De forma similar, otros estudios de bioaumentación para el tratamiento de aguas con alto contenido de proteína (ICHIDA et al., 2001; LOPERENA et al., 2009) y otros compuestos (HU et al., 2008; PARK et al., 2008; CHEN et al., 2015) han sido exitosos y demuestran la importancia de una adecuada selección de bacterias en función de los contaminantes a ser tratados.

La mayoría de los aislados bacterianos seleccionados fueron considerados como buenos productores de enzimas extracelulares ya que presentaron valores de IE entre 2,4 y 4,5 (tabla 1). Esto demuestra su potencial hidrolítico con relación a los principales compuestos presentes en este tipo de aguas residuales (proteínas, grasas y polisacáridos). No obstante, la selección de microorganismos para su aplicación en procesos de bioaumentación no solo involucra el aislamiento y la selección de bacterias con actividades metabólicas específicas. Otros aspectos que deben ser tenidos en cuenta, tales como la compatibilidad, la persistencia y la competitividad con las demás poblaciones nativas presentes en el hábitat (YU y MOHN, 2002). En ese sentido, ese efecto fue evaluado indirectamente a través de pruebas de antagonismo entre los aislados bacterianos que fueron seleccionados. De acuerdo a los resultados obtenidos, se pudo establecer que AP6, AP13, AA9 y AC4 fueron inhibidos por AA11 (tabla 2). Esto sugiere que estos aislados podrían no ser eficientes en programas de bioaumentación debido a los efectos antagónicos producidos por las poblaciones nativas presentes y/o por los propios aislados seleccionados (HERRERO Y STUCKEY, 2014).

En general, este estudio proporciona una estrategia para la selección de microorganismos degradadores de compuestos específicos como las proteínas, grasas y polisacáridos como principales constituyentes de efluentes residuales de la industria frigorífica. No obstante, futuros estudios relacionados con estrategias de bioaumentación deben ser realizados a fin de evaluar la eficiencia las $\mathrm{BH}$ obtenidas en la biodegradación de la fracción orgánica de efluentes de la industria frigorífica.

\section{Conclusión}

En este estudio fueron recuperados aislados bacterianos productores de enzimas extracelulares para la degradación de compuestos orgánicos tales como proteínas, polisacáridos y lípidos. Dichos aislados presentan un potencial promisorio para futuras investigaciones relacionadas con el desarrollo de procesos de bioaumentación, los cuales busquen optimizar la eficiencia del tratamiento de efluentes generados por industria frigorífica.

Agradecimientos: Expresamos nuestro agradecimiento a la Universidad Francisco de Paula Santander por ser el ente financiador de este proyecto, y al apoyo técnico del frigorífico "La Frontera".

\section{Referencias}

ARNOSTI, C. 2011. Microbial Extracellular Enzymes and the Marine Carbon Cycle. Annual Reviews 3:401-425.

BAZRAFSHAN, E.; MOSTAFAPOUR, F.K.; FARZADKIA, M.; OWNAGH, K.A.; MAHVI; A.H. 2012. Slaughterhouse Wastewater Treatment by Combined Chemical Coagulation and Electrocoagulation Process. Plos One 7 (8):1-8.

BEN-GIGIREY B, DE SOUSA J.M.V.B, VILLA T.G. (2000). Characterization of biogenic amine-producing Stenotrophomonas maltophilia strains isolated from white muscle of fresh and frozen albacore tuna. Int $\mathrm{J}$ Food Microbiol 57:19-31. 
CAVALEIRO, A.J.; SOUSA, D.Z.; ALVES, M.M. 2010. Methane production from oleate: assessing the bioaugmentation potential of Syntrophomonas zehnderi.water research 44 (17):4940-4947.

ÇADIRCI B.H.; ÇITAK S. 2005. A Comparison of Two Methods Used for Measuring Antagonistic Activity of Lactic Acid Bacteria. Pakistan Journal of Nutrition 4 (4): 237-241.

ČATER, M.; FANEDL, L.; MALOVRH, S.; LOGAR, R.M. 2015. Biogas production from brewery spent grain enhanced by bioaugmentation with hydrolytic anaerobic bacteria. Bioresource technology 186: 261-269.

CHAN, Y.J.; CHONG, M.F.; LAW, C.L.; HASSELL, D.G. 2009. A review on anaerobic-aerobic treatment of industrial and municipal wastewater. Chemical Engineering Journal 155:1-18.

CHEN, Q.; NI, J.; MA, T.; LIU, T.; ZHENG, M. 2015. Bioaugmentation treatment of municipal wastewater with heterotrophic-aerobic nitrogen removal bacteria in a pilot-scale SBR. Bioresource technology 183:25-32.

CUNHA, A.; ALMEIDA, A.; COELHO, F.J.R.C.; GOMES, N.C.M.; OLIVEIRA, V.; SANTOS, A.L. 2010. Bacterial extracellular enzymatic activity in globally changing aquatic ecosystems. Current research, technology and education topics in applied microbiology and microbial biotechnology. Badajoz, Spain: Formatex Research Center: 124-135.

FACCHIN, S.; ALVES, P. D.; DE FARIA SIQUEIRA, F.; BARROCA, T. M.; NETTO, J.M.; KALAPOTHAKIS, E. 2013. Biodiversity and secretion of enzymes with potential utility in wastewater treatment. Open Journal of Ecology $3(1): 34-47$

GERARDI, M.H. 2003. The microbiology of anaerobic digesters. John Wiley \& Sons. USA.

HANKIN, L.; ANAGNOSTAKIS, S. L. 1975. The use of solid media for detection of enzymes production by fungi. Mycologia 67 (3): 597-607.

HERRERO, M.; STUCKEY, D.C. 2014. Bioaugmentation and its application in wastewater treatment: A review. Chemosphere 1:10.

HU, X.; LI, A.; FAN, J.; DENG, C.; ZHANG, Q. 2008. Biotreatment of q-nitrophenol and nitrobenzene in mixed wastewater through selective bioaugmentation. Bioresour. Technol. 99: 4529-4533.

ICHIDA, J.M.; KRIZOVA, L.; LEFEVRE, C.A.; KEENER, H.M.; ELWELL, D.L.; BURTT JR., E.H. 2001. Bacterial inoculum enhances keratin degradation and biofilm formation in poultry compost. Journal of Microbiological Methods 47:199-208.

LEALEM, F.; GASHE, B. A. 1994. Amylase production by a gram-positive bacterium isolated from fermenting tef (Eraglostis tef). J. Appl. Bacteriol. 77 (3): 348352.

LEFEBVRE, X.; PAUL, E.; MAURET, M. 1998. Kinetic characterization of saponified domestic lipid residues aerobic biodegradation. Water Research 32: 3031-3038.

LIU, Y.; KANG, X., LI, X.L.; YUAN, Y. 2015. Performance of aerobic granular sludge in a sequencing batch bioreactor for slaughterhouse wastewater treatment. Bioresource technology 190:487-91

MARONE, A.,;MASSINI, G.; PATRIARCA, C.; SIGNORINI, A.; VARRONE, C.; IZZO, G. 2012. Hydrogen production from vegetable waste by bioaugmentation of indigenous fermentative communities. international journal of hydrogen energy 37 (7):5612-5622.

MARTIN-RYALS, A.; SCHIDEMAN, L.; LI, P.; WILKINSON, H.; WAGNER, R. 2015. Improving anaerobic digestion of a cellulosic waste via routine bioaugmentation with cellulolytic microorganisms. Bioresource technology 189:62-70.

MOHAN, S.V.; RAO, N.C.; PRASAD, K.K.; SARMA, P.N. 2005. Bioaugmentation of an anaerobic sequencing batch biofilm reactor (AnSBBR) with immobilized sulphate reducing bacteria (SRB) for the treatment of sulphate bearing chemical wastewater. Process Biochemistry 40 (8):2849-2857. 
MUÑOZ, D.M. 2005. Sistema de tratamiento de aguas residuales de matadero: Para una población menor 2000 habitantes [System of residual water treatment of slaughter house: For a smaller population 2000 inhabitants. Facultad de Ciencias Agropecuarias 3 (1):87-98.

PABÓN, S. L.; GÉLVEZ, J.H.S. 2009. Arranque y operación a escala real de un sistema de tratamiento de lodos activos para aguas residuales de matadero. Ingeniería e Investigación 29 (2):53-58.

PALATSI, J.; VIÑAS, M.; GUIVERNAU, M.; FERNANDEZ, B.; FLOTATS, X . 2011. Anaerobic digestion of slaughterhouse waste: Main process limitations and microbial community interactions. Bioresource Technology 102:2219-2227.

PARK, D.; LEE, D.S.; KIM, Y.M.; PARK, J.M. 2008. Bioaugmentation of cyanide-degrading microorganisms in a fullscale cokes wastewater treatment facility. Bioresour. Technol. 99:2092-2096.

TEATHER, R. M.; WOOD, P. J. 1982. Use of congo red-polysaccharide interactions in enumeration and characterization of cellulolytic bacteria from the bovine rumen. Appl. Environ. Microbiol. 43 (4):777-780.

VAN DER GAST, C.J.; WHITELEY, A.S.; THOMPSON, I.P. 2004. Temporal dynamics and degradation activity of a bacterial inoculum for treating waste metal-working fluid. Environmental Microbiology 6:254-263.

VAVILIN, A. .; FERNANDEZ, B.; PALATSI, J.; FLOTATS, X.. 2008. Hydrolysis kinetics in anaerobic degradation of particulate organic material Waste Manag. 28 (6):939-951

VIDAL, G.; CARVALHO, A.; MÉNDEZ, R.; LEMA, J.M. 2000. Influence of the content in fats and proteins on the anaerobic biodegradability of dairy wastewaters. Bioresource Technology 74:231-239.

YU, Z.T.; MOHN, W.W. 2002. Bioaugmentation with the resin-acid degrading bacteria Zoogloea resiniphila DhA-35 to counteract $\mathrm{pH}$ stress in an aerated lagoon treating pulp and paper mill effluent. Water Res. 36:2793-2801. 